\section{References}

1. Calverley PM, Boonsawat W, Cseke Z, Zhong N, Peterson S, Olsson H. Maintenance therapy with budesonide and formoterol in chronic obstructive pulmonary disease. Eur Respir $J$ 2003; 22: 912-919.

2. Szafranski W, Cukier A, Ramirez A, et al. Efficacy and safety of budesonide/formoterol in the management of chronic obstructive pulmonary disease. Eur Respir J 2003; 21: $74-81$.
3. Calverley P, Pauwels R, Vestbo J, et al. Combined salmeterol and fluticasone in the treatment of chronic obstructive pulmonary disease: a randomised controlled trial. Lancet 2003; 361: 449-456.

4. NHLBI/WHO Workshop Report, 2004. Global strategy for the diagnosis, management and prevention of chronic obstructive pulmonary disease. www.goldcopd.com. Date last accessed: July 272004.

\title{
Progressive damage on high-resolution computed tomography
}

\section{To the Editors:}

We were interested to read the article by DE JONG et al. [1]. We are writing to you following enquiries by a number of clinical colleagues who have expressed concerns regarding the regular use of a high-dose technique (i.e. high-resolution computed tomography) with groups of young patients and the implications for the substantial radiation doses that may result.

The clinical potential of the procedure has, we are sure, been clearly shown. However, given the very high radiation doses involved and the young age of these patients, there is concern that relatively little information had been given to allow adequate justification of this procedure, in accordance with the relevant European directive [2], which concerns the health protection of individuals against the dangers of ionising radiation in relation to medical exposures.

We would be interested to hear the authors' views, and, in particular, whether they are able to provide any information to allow a formal risk-benefit analysis to be carried out.

\section{Rawlings*, D. Tennant", J. Furness}

*Regional Medical Physics Dept, Newcastle General Hospital, Newcastle-upon-Tyne, "North Tyneside General Hospital, North Shields, and "Darlington Memorial Hospital, Darlington, UK.

\section{References}

1. de Jong PA, Nakano $\mathrm{Y}$, Lequin $\mathrm{MH}$, et al. Progressive damage on high-resolution computed tomography despite stable lung function in cystic fibrosis. Eur Respir $J$ 2004; 23 : 93-97.

2. European Council Directive 97/43/Euratom and repealing Directive 84/466/Euratom. The Medical Exposures Directive. europa.eu.int/comm/energy/nuclear/radioprotection/ doc/legislation/9743_en.pdf. Date last accessed: September 152004.

DOI: $10.1183 / 09031936.04 .00083304$

From the authors:

We thank D. Rawlings and colleagues for their highly relevant question. Our study [1] was performed to compare high-resolution computed tomography (HRCT) with lung function in the assessment of disease progression in cystic fibrosis $(\mathrm{CF})$. It was beyond the scope of this paper to evaluate the complex question of radiation risk versus clinical benefit of HRCT in these patients. Determining the risk-benefit profile of HRCT in CF patients is currently a research topic of high priority in our group. At this time, we believe that the use of HRCT in CF patients is consistent with the 1997 European directive [2]. In 1996, we reviewed the literature and performed a systematic review of our chest radiograph results; we concluded that routine chest radiographs were insensitive, and, due to variable techniques, we were unable to provide valid objective data on disease progression. As such, yearly bilateral chest radiographs were exposing patients to unnecessary radiation exposure. In addition, we and others concluded that lung function testing underestimated the severity and progression of lung disease in many $\mathrm{CF}$ patients. The question at that time was whether we should stop doing routine chest radiographs or introduce an examination that was undeniably more sensitive, but which provided greater radiation exposure, i.e. HRCT. Simply eliminating chest imaging would have left us unable to assess disease progression, which we believed was clinically unacceptable. CF-related lung disease results in a substantial reduction in life expectancy, substantial morbidity and requires the use of aggressive, potentially toxic and expensive therapies. For this reason, enhanced monitoring of disease progression was considered essential. In close collaboration with our radiology department, we designed the monitoring protocol that we have reported [1].

We have found that HRCT findings have allowed us to accurately estimate disease severity and tailor treatment. This conclusion has been confirmed by the retrospective analysis described in our publication [1]. We are currently extending this study with longer-term evaluation of our patients. In addition, we have compared our results to those in a cohort from a Swedish CF centre (Queen Silvia Children's Hospital, Gothenburg, Sweden) that has used a similar HRCT routine since 1997. Preliminary results from this centre are in agreement with the results we have published in the European Respiratory Journal [1].

We agree with D. Rawlings and colleagues that we should aim for the minimal possible radiation exposure that provides acceptable diagnostic information. Recent improvements in scanner technology have allowed us to reduce the radiation dose to one-tenth of our initial protocol with no substantial decrease in image quality. We believe it is likely that further scanner technical advances will allow further reduction in computed tomography radiation dose.

This response is not meant to suggest that we negate the potential risk of regular high-resolution computed tomography in cystic fibrosis children. The risk-benefit ratio for early and regular high-resolution computed tomography 
scanning must be assessed, and we are committed to ongoing analysis and re-evaluation.

\author{
P.A. de Jong*,\#, M.H. Lequin ${ }^{\top}$, J.R. Mayo ${ }^{+}$, P.D. Paré\#, \\ H.A.W.M. Tiddens* \\ Depts of *Paediatric Pulmonology, and "Paediatric Radiology, \\ Erasmus MC-Sophia, Rotterdam, The Netherlands. ${ }^{\text {\#BC }}$ \\ James Hogg iCAPTURE Centre for Cardiavascular and \\ Pulmonary Research, St Paul's Hospital, and ${ }^{+}$Dept of \\ Radiology, Vancouver Hospital and Health Science Center, \\ Vancouver, BC, Canada.
}

\section{References}

1. de Jong PA, Nakano Y, Lequin $\mathrm{MH}$, et al. Progressive damage on high-resolution computed tomography despite stable lung function in cystic fibrosis. Eur Respir $J$ 2004; 23: 93-97.

2. European Council Directive 97/43/Euratom and repealing Directive 84/466/Euratom. The Medical Exposures Directive. europa.eu.int/comm/energy/nuclear/radioprotection/ doc/legislation/9743_en.pdf.

\section{Variable phenotype associated with SP-C gene mutations: fatal case with the I73T mutation}

\section{To the Editors:}

We read with great interest the report by BRASCH et al. [1] on a case of interstitial lung disease (ILD) due to a de novo heterozygous missense mutation in the surfactant protein-C gene (SFTPC), which resulted in the substitution of threonine for isoleucine in codon 73 of the propeptide of the surfactant protein (SP)-C proprotein (I73T). Histological findings were consistent with nonspecific interstitial pneumonia (NSIP) and pulmonary alveolar proteinosis (PAP) features. Therapy included whole-lung lavages and anti-inflammatory drugs, and the child is still alive.

The natural history of lung disease associated with SP-C mutations is poorly characterised, with unpredictable shortand long-term outcomes. In order to emphasise the phenotypic variability of SFTPC mutations, we now report a child with fatal lung disease due to the SP-C I73T mutation. The child was a full-term male, born to unrelated parents from an uneventful twin dizygotic pregnancy. At 3 months, he developed episodes of asthmatic bronchitis. At 9 months, he was admitted to the hospital because of progressive failure to thrive and moderate-to-severe delay in neuromotor development that had started at 5 months. Dyspnoea and tachypnoea were observed, and arterial oxygen tension and arterial carbon dioxide tension in room air were $39 \mathrm{mmHg}$ and $37 \mathrm{mmHg}$, respectively, and $\mathrm{O}_{2}$ supplementation was started. Chest imaging findings, including conventional radiograph plus high-resolution computed tomography, were consistent with diffuse ILD. Cystic fibrosis, respiratory infectious diseases, lysinuric protein intolerance with respiratory failure due to PAP, gastro-oesophageal reflux disease, and defects in the immune system were excluded [2-4]. A thoracoscopic lung biopsy was performed when the child was 11 months old. Histology showed widened alveoli with thickened alveolar septa infiltrated mainly by fibroblasts and lymphocytes, hyperplasia of type-II pneumocytes, and massive intraalveolar accumulation of finely granular periodic acidSchiff-positive material and several cholesterol crystals, consistent with NSIP and PAP features.

An SP abnormality was suspected. Hereditary SP-B deficiency was unlikely, given the usually rapidly fatal nature of that disorder, and, moreover, immunohistochemical staining for SP-B was detected. Genetic analysis revealed a heterozygous mutation ( $\mathrm{T}$ to $\mathrm{C}$ transition in position 1286), resulting in a substitution of threonine for isoleucine (I73T) in the SFTPC. Neither parent carried this mutation, consistent with a de novo mutation resulting in sporadic disease. The mutation in the presence of ILD and the absence of the mutation and disease in the parents further strengthened the association between the genetic abnormality and the disease.

During the following 4 months, the infant often needed antibiotics because of recurrent respiratory infections, in addition to a slowly progressive need for $\mathrm{O}_{2}$ supplementation. Oral steroid treatment was started, along with evaluation for lung transplantation. Unfortunately, 3 months later, he developed acute respiratory failure associated with a possible bacterial respiratory infection, which required admission to the Paediatric Intensive Care Unit (Cardarelli Hospital, Naples, Italy). Despite mechanical ventilation, his condition progressively worsened, and the child died 8 months postbiopsy. The parents did not authorise an autopsy.

In the last few years, several mutations for the SFTPC have been identified [1, 5-10]. Recently, HAMvAs et al. [10] described an infant with a progressively severe ILD who successfully underwent lung transplantation. In this case, a spontaneous heterozygous nine base-pair deletion was identified in the exon 3 of the SFTPC, which is in the same general location in which BRASCH et al. [1] and our group demonstrated the g.1286T $>C$ transition.

Information describing the clinical expression of SFTPC mutations is accumulating, and it is clear that such mutations can cause ILD in children and adults. The histological expression of mutations associated with SFTPC is pleiotropic, and ranges from chronic pneumonitis of infancy and nonspecific ILD in children to usual or desquamative interstitial pneumonia and idiopathic pulmonary fibrosis in adults [11]. Moreover, age at presentation is quite variable, with some children developing symptoms in early infancy, and others not until adulthood, and some remaining symptom free. As the same specific mutations can be associated with variable phenotypic expression, modifier genes or interactions between genetic background and environmental factors are likely to be important in modifying the course of the disease $[8,9]$.

Further specific information about clinical manifestations, histological appearance and genetic basis related to surfactant protein-C abnormalities are needed in order to provide an explanation for familial and sporadic cases of interstitial lung disease that may remain otherwise undiagnosed. Since the clinical course may be characterised by periods of substantial improvement, followed by others with more severe activity triggered by infections eventually leading to fatal respiratory failure, a better understanding of what modifies or exacerbates the course of this disease might provide some valuable clues on how to better treat it. Finally, given the variability of phenotypic expression of the lung disease related to surfactant 